\title{
Row Replacement Algorithms for Screen Editors
}

\author{
EUGENE W. MYERS \\ University of Arizona \\ and
}

WEBB MILLER

The Pennsylvania State University

\begin{abstract}
Interactive screen editors repeatedly determine terminal command scquences to update a screen row. Computing an optimal command sequence differs from the traditional sequence comparison problem in that there is a cost for moving the cursor over unedited characters and the cost of an $n$-character command is not always the cost of $n$ one-character commands. For example, on an ANSI-standard terminal, it takes nine bytes to insert one character, ten to insert two, eleven to insert three, and so on. This paper presents an $O(M N)$ dynamic programming algorithm for row replacement where an $n$-character command costs $\alpha n+\beta$ for constants $\alpha$ and $\beta$. $M$ is the length of the original row and $N$ is the length of its replacement. Also given is an $O(\operatorname{Cost} \times(M+N))$ "greedy" algorithm for optimal row replacement. Here Cost is the optimal cost (in bytes) of the replacement, so the algorithm is fast when the required update is small. Though the algorithm is rather complicated, it is fast enough to be useful in practice.
\end{abstract}

Categories and Subject Descriptors: F.2.2 [Analysis of Algorithms and Program Complexity]: Nonnumerical Algorithms and Problems-computations on discrete structures

General Terms: Algorithms

Additional Key Words and Phrases: Dynamic programming, greedy algorithm, row replacement, screen editor

\section{THE ROW REPLACEMENT PROBLEM}

Screen-oriented programs maintain a representation of an object and present a view of it on the screen. For example, screen editors keep an internal edit buffer and display a block of lines from the buffer. The screen must be updated when the object is changed. In one solution, procedures that modify the object must also update the view or at least specify how the view has changed. Optimal use of such a package [1] may require learning and calling many different routines. A cleaner approach lets an autonomous screen manager module determine how

This work was supported in part by National Science Foundation grant DCR-8511455.

Authors' current addresses: E. W. Myers, Department of Computer Science, University of Arizona, Tucson, AZ 85721; W. Miller, Department of Computer Science, The Pennsylvania State University, University Park, PA 16802.

Permission to copy without fee all or part of this material is granted provided that the copies are not made or distributed for direct commercial advantage, the ACM copyright notice and the title of the publication and its date appear, and notice is given that copying is by permission of the Association for Computing Machinery. To copy otherwise, or to republish, requires a fee and/or specific permission.

(C) 1989 ACM 0164-0925/89/0100-0033 $\$ 01.50$ 
to update the screen by comparing its record of screen contents with views of the modified object. The interface to the screen manager is then a single routine, refresh, that updates the screen with respect to the current object. It is given no information other than the object and screen contents. Writers of screen editors are almost unanimous in recommending the use of an autonomous screen manager $[2,6,10,18,24]$. The design greatly improves the editor's internal structure, so it is appropriate with both ASCII and bitmap displays.

Care is needed to achieve satisfactory efficiency with an autonomous screen manager. In many contexts, data travels from the editor to the screen at between 100 and 1,000 characters per second (e.g., 1,200 or 9,600 baud), which is orders of magnitude slower than the editor's computing speed. Under such circumstances, it is worthwhile for the editor to compute a minimal, or near minimal, set of row-updating commands, as long as the time to do so does not outweigh the savings in character-transmission time. Although these considerations are far less important with high-speed communication and bitmap displays, the results of this paper will be useful as long as people want to access remote processors over slow transmission media, such as telephone lines.

For autonomous screen managers, a basic problem is to optimally update a screen row. An algorithm, presented with the row currently on the screen and the desired row contents, must produce a shortest-possible sequence of terminal commands that replaces the existing row with the desired row. This row replacement problem is complicated by varying terminal capabilities and command encoding schemes. This paper assumes the following terminal operations, which are provided by most modern terminals.

-Clear. The characters at, and to the right of, the cursor are deleted. The cursor does not move.

-Delete. The character at the cursor is deleted, causing later characters to be shifted left. The cursor does not move, so it ends up on the character that followed the deleted character.

-Insert $x$. The character $x$ is inserted at the cursor's location, causing the characters at and right of the cursor to be shifted to the right. The cursor moves one position to the right, so it stays with the same character.

-Move to $k$. The cursor is moved to the $k$ th column in the current row.

-Replace by $x$. The character $x$ is displayed at the cursor's location, overwriting the previously displayed character. The cursor moves one position to the right.

Command encodings vary widely among terminal brands. Typically, replace is performed by simply sending the character to the terminal. Other commands contain escape sequences that encode the operator. Table I gives escape sequences for ANSI-standard terminals and for the IBM 3101 where $\langle e s c\rangle$ denotes the "escape character". The clear and delete commands are three bytes long in one case, two in the other. With ANSI-standard terminals, a string of characters is inserted by sending a 4-byte sequence to put the terminal in insert mode, then sending the desired characters, and finally sending a 4-byte sequence to exit from insert mode. For the 3101 , each character must be inserted individually using a 3 -byte escape sequence. Thus, inserting $n$ consecutive characters costs $n+8$ bytes in one case and $3 n$ bytes in the other.

ACM Transactions on Programming Languages and Systems, Vol. 11, No. 1, January 1989. 
Table I. Two Command Encoding Schemes

\begin{tabular}{lcc}
\hline \multicolumn{1}{r}{ Instruction } & ANSI standard & IBM 3101 \\
\hline clear & $\langle e s c\rangle[K$ & $\langle e s c\rangle I$ \\
delete & $\langle e s c\rangle[P$ & $\langle e s c\rangle Q$ \\
enter insert mode & $\langle e s c\rangle[4 h$ & - \\
insert character & - & $\langle e s c\rangle P\langle$ char $\rangle$ \\
leave insert mode & $\langle e s c\rangle[4 l$ & - \\
\hline
\end{tabular}

On ANSI-standard terminals, the cursor is moved by the command ' $\langle$ esc $\rangle[\alpha ; \beta H$ ' where $\alpha$ is the decimal representation of the row number and $\beta$ is the column number. For example the 7-byte command ' $\langle$ esc $\rangle[5 ; 20 \mathrm{H}$ ' moves to row 5, column 20. For these terminals, the move command is 6 , 7, or 8 bytes long, depending on the destination (and assuming less than 100 rows and columns). The 3101 uses the 4-byte move command ' $\langle e s c\rangle \mathrm{Y} \rho \gamma$ '. Here $\rho$ is a byte containing 31 plus the row number, and $\gamma$ is 31 plus the column number. The designers add 31 because $\rho$ and $\gamma$ are then printable characters in the ASCII code.

Implementation of a row-replacement strategy requires that a number of additional details be addressed. With a line whose length exceeds the screen width, some editors fold the line onto several screen rows to make all characters visible. Other editors add a special character at the right margin of the screen to indicate an overlength line. In either case, a side effect of inserting a character in a long line is to delete the rightmost character in the row by pushing it off the screen. The implementation of a screen-update procedure must also be concerned about swamping the terminal by sending control sequences too quickly; "pad characters" or "handshaking" may be required. Such implementation details are not considered in this paper.

The commands discussed above are used for intraline editing or, as we call it, row replacement. Other commands, like delete row and insert row, manipulate entire screen rows. The methods used by screen editors to recycle entire rows are often surprisingly different from those for reusing parts of a row. A complete description of one design is given in [10, pp. 280-316], where the row-replacement strategy simply avoids retransmission of a common prefix and, if it saves time, of a common suffix. This paper is concerned only with row replacement, though methods for interline editing are mentioned briefly in the remainder of this section and under "Open Problems" in Section 5.

Gosling [6] proposes a rigorous approach to screen updating based on an offthe-shelf application of a sequence-comparison algorithm for both intra- and interline edits. However his comparison model ignores cursor-movement costs and charges an $n$-symbol operation like $n$ one-symbol operations. Moreover efficiency considerations lead him to use the length of $B$ as an approximation to the cost of replacing row $A$ by $B$ when evaluating possible interline editing scripts. With these approximate costs, the classic dynamic programming algorithm is quite practical for interline edits. Indeed Gosling used the approach in the UNIX EMACS editor, though row replacement is nonoptimally performed by simply retaining common prefixes and suffixes of the old and new lines. The resulting screen manager is quite independent of the remainder of the editor, as illustrated by the use of Gosling's code in the Maryland Window System [23]. 
Myers [15] uses sequence comparison techniques to solve the "window positioning problem": find a screen-sized block of buffer lines that contains the current line and reuses the maximum number of currently visible lines. If $R$ is the number of screen rows, then there are generally $R$ choices for the window position. A straightforward solution requires time $O\left(R^{3}\right)$ when only interline edits are considered, but Myers' algorithm needs only time $O\left(R^{2}\right)$. Miller and Myers [13] give another optimal row-replacement algorithm with worst-cost complexity $O\left(M^{2} N^{2}\right)$, but which works efficiently for many practical screenupdating problems.

This paper presents two worst-case efficient algorithms for optimal row replacement. That is, under certain assumptions about the terminal and the permissible scripts of screen-update commands, these algorithms minimize the number of bytes sent to the screen. Section 2 places these algorithms in historical perspective by sketching earlier work on the related sequence-comparison problem. Although sequence-comparison algorithms are not immediately applicable to row replacement, as shown in Section 2, they contain the seeds of our rowreplacement algorithms. Sections 3 and 4 give $O(M N)$ and $O(\operatorname{Cost} \times(M+N))$ row-replacement algorithms, respectively. Section 5 concludes with further refinements, empirical results, and open problems. The experiments cited in Section 5 show that the $O(\operatorname{Cost} \times(M+N))$ row-replacement algorithm is fast enough to be of practical value.

\section{SEQUENCE COMPARISON ALGORITHMS}

Sequence-comparison problems arise in a variety of disciplines, including computer science and molecular biology [21]. The basic problem can be phrased in terms of editing operations as follows. Given sequences $A=a_{1} a_{2} \cdots a_{M}$ and $B=$ $b_{1} b_{2} \cdots b_{N}$, determine a sequence of "basic operations" that converts $A$ to $B$ and minimizes the sum of the operations' costs (or "weights"). Traditionally, the only operations permitted are Delete, Insert, and Replace. There is no notion of a cursor; an operation may be applied at any point in the string. Moreover the cost of an operation does not depend on its context in the edit script.

In the point indel model, the basic operations insert a single symbol, delete a symbol, or replace one symbol by another, and an operation's cost may depend on the affected symbol or symbols:

$\begin{array}{ll}d_{-} \operatorname{cost}(a) & \text { cost of deleting } a \\ i_{-} \operatorname{cost}(b) & \text { cost of inserting } b \\ r_{-} \operatorname{cost}(a, b) & \text { cost of replacing } a \text { with } b \text { when } a \neq b\end{array}$

In the block indel model, an indel (i.e., insertion or deletion) of $k>0$ consecutive symbols is treated as an atomic operation, rather than $k$ operations on individual symbols. The operation is assigned a weight, or gap penalty, $w_{k}$ that depends only on the number of symbols inserted or deleted. A variation of the model allows insertion costs to differ from deletion costs. The block indel model also includes a single-symbol Replace operation of cost $r_{-} \operatorname{cost}(a, b)$.

Simple dynamic programming algorithms compute optimal edit scripts for the point indel problem in time $O(M N)$ [20] and for the block indel problem in time $O(M N(M+N))$ [22], where $M$ and $N$ are the sequence lengths. Let $A_{k}$ denote 
Table II. $C(i, j)$ for $A=a b c a b b a, B=c b a b a c$

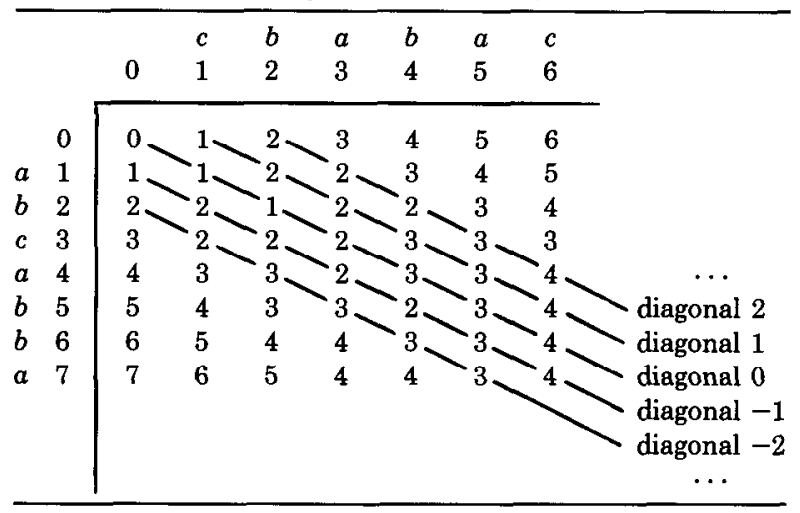

the $k$-symbol prefix, $a_{1} a_{2} \ldots a_{k}$, of $A$. A dynamic programming approach for finding the minimum cost of editing $\mathrm{A}$ into $\mathrm{B}$ requires computing $C(i, j)$, the minimum cost of editing $A_{i}$ into $B_{j}$ for all $i \in[0, M]$ and $j \in[0, N]$. For example, if $A$ is abcabba, $B$ is cbabac, and we allow single-symbol Delete, Insert, and Replace operations of cost 1 , then $C$ is shown in Table II. Observe that $C(4,4)=3$ since $A_{4}=a b c a$ can be edited to $B_{4}=c b a b$ with either the sequence 'Replace $a_{1}$ with $b_{1}$, Delete $a_{3}$, Insert $b_{4}$ after $a_{4}$ ' or the sequence 'Replace $a_{1}$ with $b_{1}$, Replace $a_{3}$ with $b_{3}$, Replace $a_{4}$ with $b_{4}^{\prime}$. Notice there is no cost associated with shifting attention from one part of the string to another and commands may be applied in any order.

The following $O(M N)$ dynamic programming algorithm [20] computes $C$ for the point indel model.

Algorithm 1a. Computing $C(i, j)$.

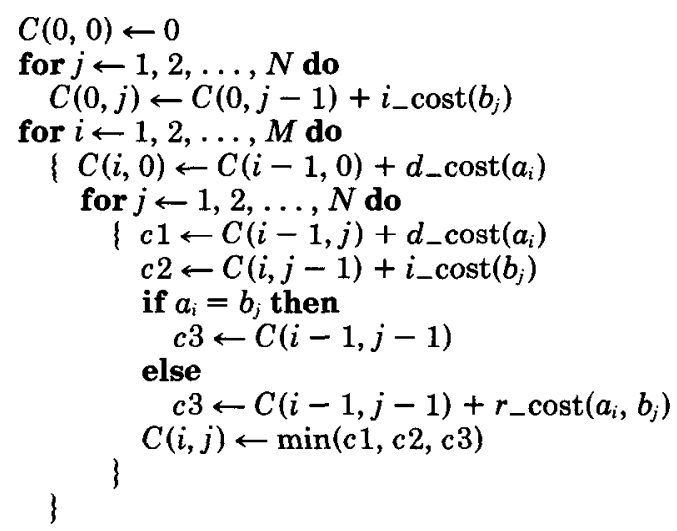

The correctness of the algorithm can be seen by considering the computation of $C(i, j)$ in the innermost loop. Any edit script converting $A_{i}$ into $B_{j}$ must either (1) delete $a_{i},(2)$ insert $b_{j}$, or (3) replace $a_{i}$ by $b_{j}$ if $a_{i} \neq b_{j}$. If the script is optimal, then the remainder of the script optimally solves a subproblem. In case 1 , the script consists of an optimal script converting $A_{i-1}$ to $B_{j}$ plus the delete command. In case 2 , it consists of an optimal script converting $A_{i}$ to $B_{j-1}$ plus the insert 
command. In case 3 , it consists of an optimal script converting $A_{i-1}$ to $B_{j-1}$ plus the replace command if necessary. The algorithm considers the three forms of edit scripts, and selects the best.

Notice that extending an edit script by adding a delete operation corresponds to a vertical move in the table from the $(i-1, j)$ grid points to $(i, j)$. Extensions by insert and replace operations correspond to horizontal and diagonal moves, respectively. The alignment of $a_{i}$ with $b_{j}$ when $a_{i}=b_{j}$ corresponds to a diagonal zero-cost move to grid point $(i, j)$. A script for editing $A$ to $B$ corresponds to a sequence or path of moves from point $(0,0)$ to point $(M, N)$. The cost of a script is the sum of the costs of the individual moves. Thus finding a minimum-cost script corresponds to finding a minimum-cost or shortest path from $(0,0)$ to $(M, N)$.

The insight that string editing is a special instance of the shortest paths problem led to the design of faster "greedy" algorithms for the point indel problem where all operations cost $1[11,14,19]$. Let diagonal $k$ consist of those grid points $(i, j)$ for which $i+k=j$, as shown in Table II. The diagonals of the $C$ matrix range from $-M$ to $N$. Let $L(c, k)$ be the length, $i$, of the longest prefix $A_{i}$ that can be converted to $B_{i+k}$ with a script of cost $c$. If no such prefix exists, define $L(c, k)$ to be -1 . Because all costs are 1 , it follows that the values in the $C$ matrix are nondecreasing along diagonals and increase by at most 1 with each diagonal move. Thus, for this special problem, one can prove that $L(c, k)$ gives the row containing the last $c$ in diagonal $k$ if such a row exists. For example, $L(3,1)=4$ and $L(1,3)=-1$. The reader should note that this characterization of $L$ values in terms of $C$ values will not suffice for the more general greedy algorithm of Section 4.

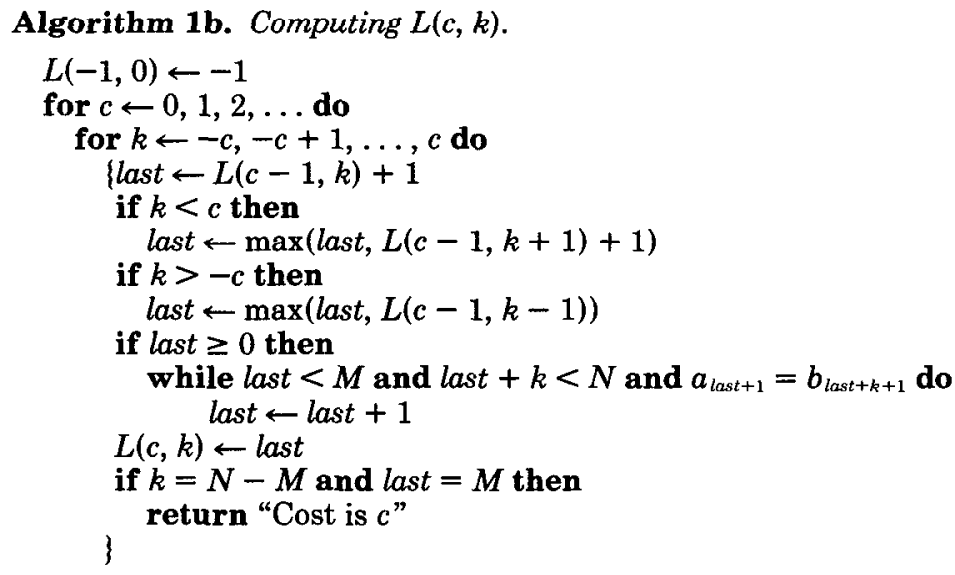

Greedy algorithms fill in $L$ for $c=0,1,2, \ldots$, until the lower, right-hand value of $C$ is known, i.e., they find the value $c$ for which $L(c, N-M)=M$. Informally, the logic for computing $L(c, k)$ goes as follows. We are looking for a $c$ that is as far down diagonal $k$ as possible. A script that costs $c$ consists of a script of cost $c-1$ plus another operation. If this final operation is delete, then the best we can do is to make a vertical move from the last $c-1$ on diagonal $k+1$; this reaches row $L(c-1, k+1)+1$. If the final operation is insert, row $L(c-1, k-$ 1) can be reached by a horizontal move from the last $c-1$ on diagonal $k-1$. If 
the final operation is replace, row $L(c-1, k)+1$ can be reached with a diagonal move. From whichever move places us in the greatest row, last, we then follow zero-cost diagonal moves $\left(a_{\text {last }+1}=b_{\text {last }+k+1}\right)$ until the last $c$ entry is reached.

The central advantage of the greedy approach is that for a given $c, L$-values need to be computed only for $k$ between $-c$ and $c$. Any script leading to diagonal $k$ must involve $k$ insert operations if $k$ is positive or $k$ delete operations if $k$ is negative, and hence the script must have cost $k$ or greater. Thus, for $k \notin[-c, c]$, we know that $L(c, k)=-1$ and hence may ignore such entries.

Suppose the minimum cost of editing $A$ to $B$ is Cost. The code within the two for loops is repeated $O\left(\mathrm{Cost}^{2}\right)$ times since the outer loop is repeated Cost +1 times and the inner loop is repeated $2 c+1$ times. Thus the algorithm consumes $O\left(\operatorname{Cost}^{2}\right)$ time with the exception of the while loop, which must be accounted for separately. Charge each iteration of this loop to the grid point (last +1 , last $+k+1)$ for which the character alignment occurs. Each grid point is charged at most once and all grid points processed are between diagonals -Cost and Cost. Thus $O($ Cost $\times(M+N))$ time is spent in this loop, the dominant cost of the algorithm.

The $O(\operatorname{Cost} \times(M+N))$ worst-case time bound tells only part of the story behind the superiority of the greedy approach. Indeed, there are other sequence comparison algorithms that achieve this performance [4, 19] and work for arbitrary (noninteger) costs. However, the greedy algorithm's expected performance is often far better than this bound indicates since not all entires of a given cost $c$ are explicitly found. For example, when $r_{-} \operatorname{cost}(a, b)=\infty$ (i.e., the longest common subsequence problem) the greedy algorithm has expected running time $O\left(N+\operatorname{Cost}^{2}\right)[14]$.

Algorithms 1a and $1 b$ determine only the minimum cost; they do not explicitly produce a shortest-possible edit script. Construction of a script is covered elsewhere $[11,14,20]$. It is easy to economize the use of space in both the dynamic programming and the greedy algorithms for the minimum cost; both require $O(M+N)$ space. It is possible $[9,14]$, though more difficult, to construct edit scripts in $O(M+N)$ space.

The dynamic programming and greedy algorithms developed above work only for the point indel model. For applications where the block indel model is appropriate, it is critical to find natural conditions on the gap weights $w_{k}$ that permit the problem to be solved in time close to $O(M N)$, since the $O(M N(M+N))$ time for general weights is often prohibitive. A natural restriction is that $w_{k}$ be concave:

$$
\Delta w_{k} \geq \Delta w_{k+1} \quad \text { for all } k \geq 1, \quad \text { where } \Delta w_{k}=w_{k+1}-w_{k}
$$

In words, $w$ is concave if the cost of inserting or deleting an additional symbol decreases with the size of the affected substring. For concave weighting functions, the dynamic programming approach can be modified to run in time $O(M N \log (M+N))$ [12]. Affine gap penalties, i.e., the further restriction that $w_{k}=\alpha+\beta k$ for constants $\alpha, \beta$, permit a solution in time $O(M N)$ [7]. Affine gap costs are currently preferred in biological applications [5], though more general concave weights have also been advocated.

The block indel model with affine indel costs is appropriate for screen update costs, such as the $(8+n)$-byte cost of inserting $n$ characters on an ANSI-standard 
terminal. To see why the point indel would produce anomalous results, consider replacing row ' $a b x \ldots$...' with row ' $y a b a b z$...'. If inserting $n$ characters costs $I n$ for some constant $I$, then the command sequence, 'Insert $y$, Move to 4, Insert $a b$, Replace by $z$ ', costs the same as 'Insert yab, Move to 6, Replace by $z$ '. However, with ANSI-standard terminals, the first command set costs 8 bytes more than the second (assuming that move is not permitted in insert mode).

While the algorithmic paradigms of traditional sequence comparison algorithms are relevant to row replacement, these algorithms do not immediately solve the row-replacement problem. A major shortcoming is that the string edit models ignore the cost of cursor movement. For example, consider the problem of replacing row 'xaxaxaxax' with 'yayayayay'. On typical terminals, repeatedly moving the cursor to the next $x$ and replacing it by $y$ is inferior to simply replacing every character. The clear operation is also omitted from the edit models. For updating screen row 'repeated repeated' to the string 'repeated', moving the cursor to the space between the two words and clearing the row is less expensive on an ANSI-standard terminal than nine delete commands.

\section{A DYNAMIC PROGRAMMING ALGORITHM FOR ROW REPLACEMENT}

Let OPS be the set \{delete, insert, move, replace\} and let OPS+ be the full operation repertoire, OPS $\cup\{$ clear $\}$. In the row-replacement framework, the cost of $n$ consecutive op commands is assumed to be startup(op) $+n \times$ perchar (op) where $\operatorname{startup}(o p)$ and perchar $(o p)$ are nonnegative integers modeling the startup and per-character costs for op. For example, Table III gives cost parameters for ANSI-standard terminals. The startup cost is paid (1) for the first operation of an edit script, and (2) whenever an operation differs from the previous operation. Thus, 'Replace by a, Move to 4, Insert xy' costs startup(replace) $+1 \times$ per$\operatorname{char}($ replace $)+\operatorname{startup}($ move $)+2 \times$ perchar $($ move $)+\operatorname{startup}($ insert $)+2 \times$ perchar(insert) $=19$ for ANSI-standard terminals. The coefficient of perchar (move) is 2 because the cursor is moved two columns to the right.

This cost accounting is a simplification. For example, it does not model the variable cost of 6-8 bytes for an ANSI-standard move command. (We assume conservatively that move costs 8 bytes.) Moreover some terminal brands allow cursor movement, deleting of characters, etc., while in "insert mode," so this approach may overestimate attainable costs by charging for unnecessary startups. However, the astute reader will observe that our row-replacement algorithms can be adapted to cover such variations.

The algorithms developed in this section and the next compute a minimumcost left-to-right conversion of $A$ to $B$. A conversion is left-to-right if move operations always move the cursor one or more columns to the right of its current position. If the cursor starts in column 1 and characters are not "spilled" off the end of the row, then a minimum-cost left-to-right script is guaranteed to be optimal over all row-updating scripts. Otherwise near-optimal scripts can be obtained from our left-to-right scripts as follows. If the cursor is not initially in position 1, then preface the script with 'Move 1'. If a spill occurs, then append commands that move to the end of the line and restore the spilled characters. Alternatively our algorithms can be modified to consider only left-to-right scripts that avoid spills. To do this, entries of the cost matrix above diagonal $W-M$ are ignored, where $W$ is the screen width and $M$ is $A$ 's length. 
Table III. ANSI Standard Command Costs

\begin{tabular}{lcc}
\hline & startup & perchar \\
\hline clear & 3 & 0 \\
delete & 0 & 3 \\
insert & 8 & 1 \\
move & 8 & 0 \\
replace & 0 & 1 \\
\hline
\end{tabular}

Table IV. $C(i, j, o p)$ for $A=a b c a b b a, B=c b a b a c$

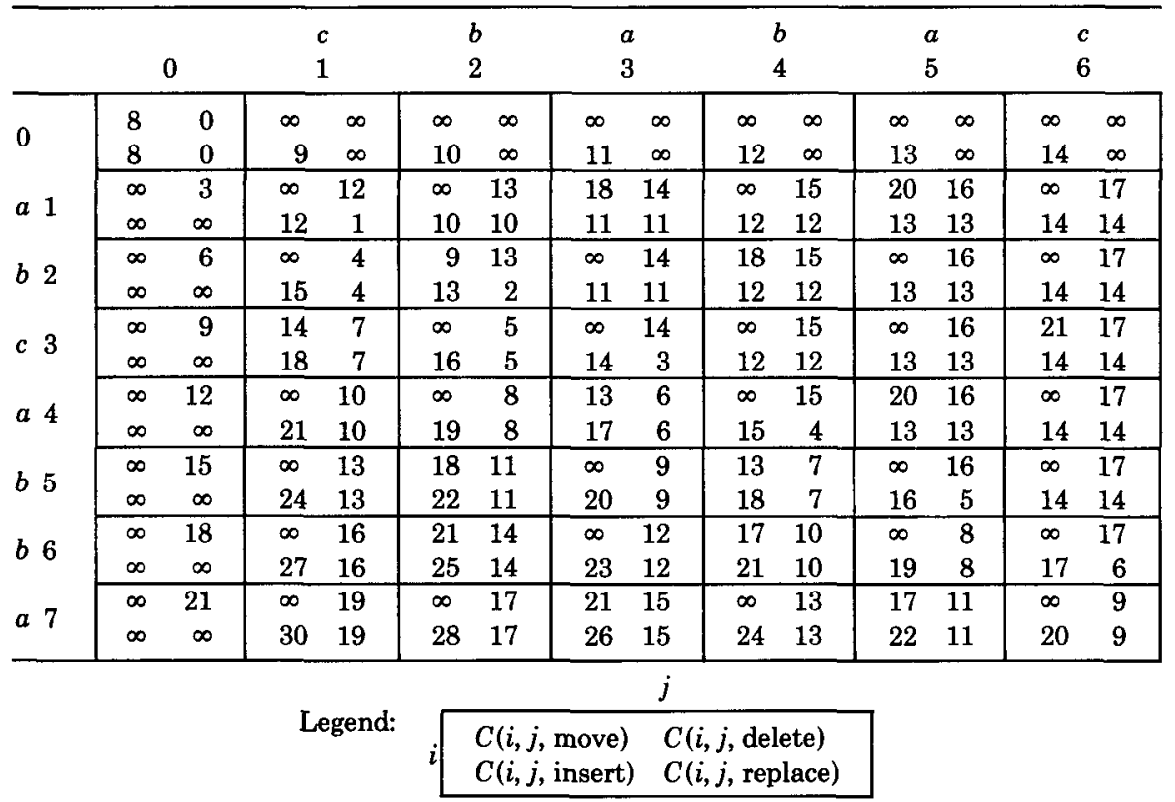

The basic row-replacement algorithm operates in two steps. First a dynamicprogramming algorithm fills in a table giving minimum costs for row replacement using certain restricted kinds of edit scripts. In a second pass, boundary values in the table are inspected to account for operations omitted from the first step.

A restricted replacement script for $A_{i}=a_{1} a_{2} \cdots a_{i}$ and $B_{j}=b_{1} b_{2} \cdots b_{j}$ is a leftto-right script that satisfies:

(1) The cursor ends in column $j+1$,

(2) clear is not used, and

(3) replace commands are not permitted once the cursor is beyond the end of the current string.

One should think of $a_{1} a_{2} \ldots a_{i}$ as terminated by a "null character" that is carried along as the edit operations are performed. The cursor ends up positioned on it. With restricted replacement scripts, one may insert at the null character or move to it, but it may not be deleted or replaced.

For each $i \leq M, j \leq N$, and op $\in O P S$, define $C(i, j, o p)$ to be the minimum cost over all restricted replacement scripts for $A_{i}$ and $B_{j}$ whose last operation is $o p$. Define $C(i, j, o p)$ to be $\infty$ if no such script exists. Table IV gives $C(i, j, o p)$ for 
the problem $A=a b c a b b a$ and $B=$ cbabac under the ANSI-standard cost model. For example, $C(3,2$, delete $)=5$ because 'Replace by $c b$, Delete' is a restricted replacement script for $a b c$ and $c b$ that is optimal among those ending with delete. Moreover, since $a_{3}$ differs from $b_{2}$, no restricted replacement script converting $a b c$ to $c b$ ends with move; thus, $C(3,2$, move $)=\infty$.

Reasoning similar to that for the dynamic programming algorithm of Section 2 leads to the following $O(M N)$ algorithm for computing $C(i, j, o p)$ for all choices of $i, j$, and op. This algorithm and the next use the definition:

$$
\operatorname{onechar}\left(o p_{1}, o p_{2}\right)= \begin{cases}\operatorname{perchar}\left(o p_{2}\right) & \text { if } o p_{1}=o p_{2} \\ \operatorname{startup}\left(o p_{2}\right)+\operatorname{perchar}\left(o p_{2}\right) & \text { if } o p_{1} \neq o p_{2}\end{cases}
$$

Onechar $\left(o p_{1}, o p_{2}\right)$ is the cost of applying $o p_{2}$ to a character if the previous operation was $o p_{1}$.

Algorithm 2a. Computing $C(i, j, o p)$.

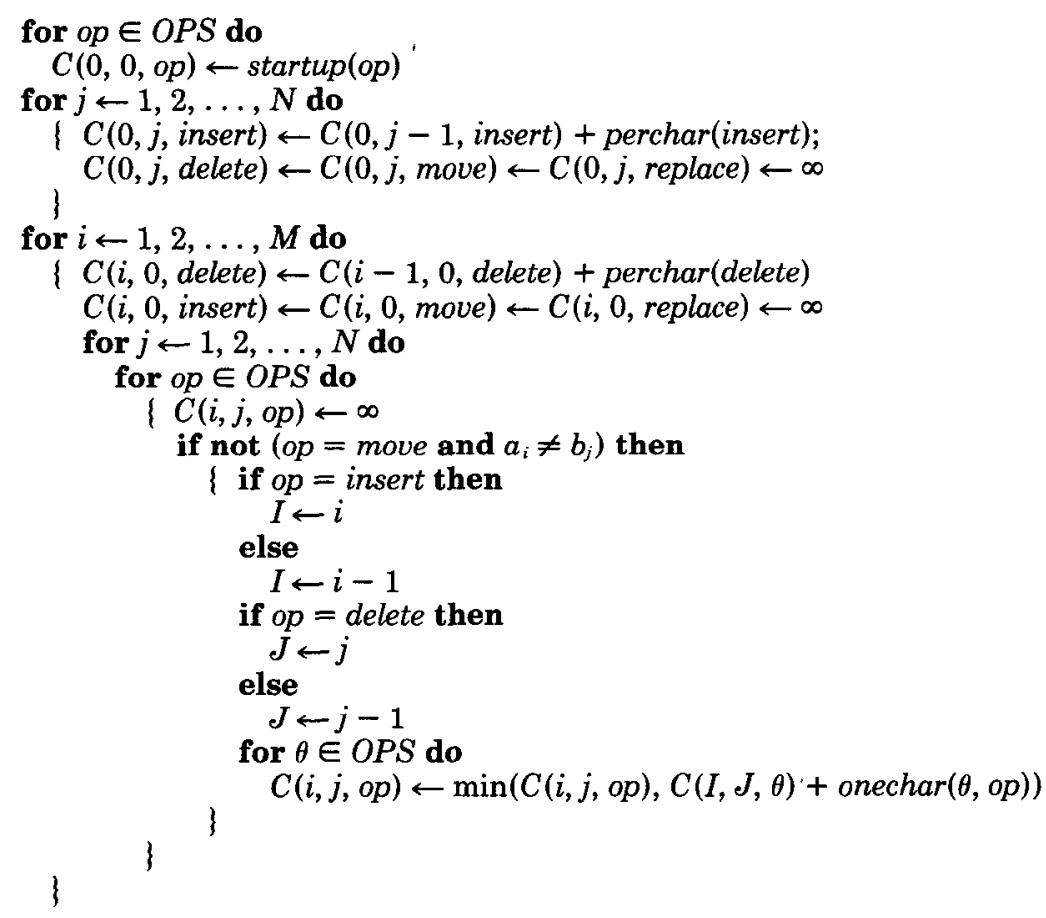

$C(0,0, o p)$ is initialized to $\operatorname{startup}(o p)$ so that the first operation in a script is charged a startup cost. Values in row 0 give costs for converting the empty string to $B_{j}$. With restricted replacement scripts this conversion requires $j$ insert operations, so for $j>0$ :

$$
\begin{gathered}
C(0, j, \text { insert })=\operatorname{startup}(\text { insert })+j \times \text { perchar (insert }) \\
C(0, j, \text { op })=\infty \text { for op } \in\{\text { delete, replace, move }\}
\end{gathered}
$$

Similarly, converting $A_{i}$ for $i>0$ to the empty string requires $i$ delete operations, so:

$$
\begin{gathered}
C(i, 0, \text { delete })=\operatorname{startup}(\text { delete })+i \times \text { perchar }(\text { delete }) \\
C(i, 0, \text { op })=\infty \text { for op } \in\{\text { insert }, \text { replace, move }\}
\end{gathered}
$$


Consider the computation of $C(i, j$, delete $)$ where $i>0$ and $j>0$. We want the optimal cost of a restricted replacement script converting $A_{i}$ to $B_{j}$ that ends with delete. If the delete is removed, then the reduced script optimally converts $A_{i-1}$ to $B_{j}$ and its length is one of the values $C(i-1, j, \theta)$ where $\theta \in$ OPS. It then follows that

$$
C(i, j, \text { delete })=\min _{\theta \in O P S}(C(i-1, j, \theta)+\text { onechar }(\theta, \text { delete }))
$$

Similar reasoning verifies the remaining recurrences that justify the algorithm:

$$
\begin{aligned}
& C(i, j, \text { insert })=\min _{\theta \in O P S}(C(i, j-1, \theta)+\text { onechar }(\theta, \text { insert })) \\
& C(i, j, \text { replace })=\min _{\theta \in O P S}(C(i-1, j-1, \theta)+\operatorname{onechar}(\theta, \text { replace })) \\
& C(i, j, \text { move })=\text { if } a_{i} \neq b_{j} \text { then } \infty \\
& \text { else } \min _{\theta \in O P S}(C(i-1, j-1, \theta)+\text { onechar }(\theta, \text { move }))
\end{aligned}
$$

Once $C$ has been computed, more general row-replacement scripts can be considered. First, move operations can be removed from the ends of scripts since they do not help transform $A$ to $B$. Second, clear commands can be utilized. Third, instead of inserting characters at the right end of $B$, the replace operation can be used to simply write them. Additional improvements are possible, but are not considered here. For example it might be preferable to delete the last character of $A$ by replacing it by a blank.

The following $O(M+N)$ second pass examines the array $C$ produced in the first step in order to treat more general replacement scripts. $\operatorname{Min} 4(i, j)$ is assumed to return the minimum of $C(i, j, o p)$ over op $\in O P S$.

Algorithm 2b. Optimizations after $C$ has been computed.

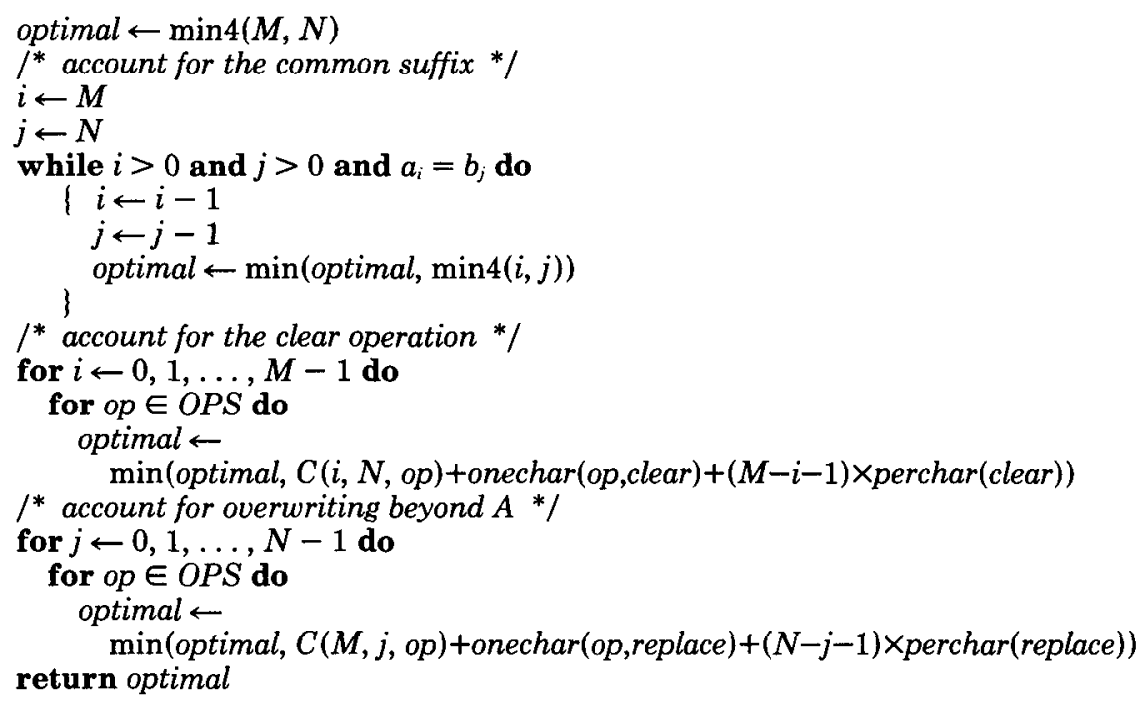

These optimizations are based on the following observations:

(1) Suppose $a_{i+1} a_{i+2} \cdots a_{M}=b_{j+1} b_{j+2} \cdots b_{N}$ and $S$ is a restricted replacement script converting $A_{i}$ to $B_{j}$. Then $S$ converts $A$ to $B$ (and leaves the cursor 
on $\left.b_{j+1}\right)$. Note that a common suffix is not necessarily preserved by an optimal script, and hence may not be automatically stripped from the original problem.

(2) It can be assumed without loss of generality that clear, if it appears in an optimal replacement script, is the final operation. This is because (a) a clear or delete after a clear is meaningless; (b) move (to the right) after a clear is also useless since blanks are not treated specially; and (c) a clear-insert or clear-replace pair is equally well performed by an insert-clear or a replaceclear, respectively. Suppose $S$ is a restricted replacement script converting $A_{i}$ to $B$. Appending a clear operation to $S$ gives a script that converts $A$ to $B$ at cost $\operatorname{cost}(S)+\operatorname{startup}($ clear $)+(M-i) \times$ perchar $($ clear $)$.

(3) Suppose $A$ is converted to $B_{j}$ at cost $C(M, j, o p)$ by a restricted replacement script that ends with the operation op. The script positions the cursor just after $b_{j}$, so simply replacing by $b_{j+1} b_{j+2} \cdots b_{n}$ produces $B$ and the augmented script costs $C(M, j, o p)+$ onechar $(o p$, replace $)+(N-j-1) \times$ perchar(replace).

It takes only $O(M+N)$ additional time to produce a minimum-cost replacement script once the cost matrix $C$ is computed. First determine the entry $C(i, j$, $o p)$ from which optimal's value was attained in the post-pass and output as the last command 'clear', 'Replace by $b_{j+1} \cdots b_{N}$ ', or nothing, as is appropriate. Then trace a path of vertical, horizontal, and diagonal moves backwards from $C(i, j$, $o p)$ to $C\left(0,0, o p^{\prime}\right)$ such that the value at the end of each move is the value at the start plus the onechar cost of the move. Each move to entry $C(i, j, o p)$ corresponds to a command: $C(i, j$, insert $)$ to 'Insert $a_{i}$ ', $C(i, j$, delete $)$ to 'Delete', $C(i, j$, replace $)$ to 'Replace by $b_{j}$ ', and $C(i, j$, move $)$ to 'Move to $j+1$ '. Listing these commands as the path is traversed in the forward direction gives the restricted portion of the script.

\section{A GREEDY ALGORITHM FOR ROW REPLACEMENT}

The greedy algorithm considers, for successive values of $c$, prefixes of row $A$ that can be edited into a prefix of row $B$ at cost $c$. Suppose the minimum cost of replacing $A$ with $B$ is Cost. The greedy algorithm examines only $O(\operatorname{Cost} \times(M+N))$ grid points of the cost matrix $C$ of Section 3, so it is faster than the dynamic programming algorithm when Cost is small.

The greedy algorithm's complexity is reduced by permitting left-to-right scripts to include move operations that leave the cursor in its current position. However no optimal script contains such commands, so reported answers are unaffected. This relaxation was not considered in Section 3 because it unnecessarily complicates the computation of $C$.

For given integers $c$ and $k$ and $o p \in O P S$, let $L(c, k, o p)$ be the length, $i$, of the longest prefix $A_{i}$ that can be converted to $B_{i+k}$ by a restricted replacement script of cost $c$ that ends with operation op. If no such prefix exists, then let $L(c, k, o p)$ equal $N O N E$, a negative number, say -1 . The relationship between $L$ and the matrix $C$ of Section 3 can be captured using the correspondence between edit scripts and paths sketched in Section 2. Computing $C$ corresponds to finding, for each grid point $(i, j)$ and operation $o p$, the cost of a shortest path from $(0,0)$ to 
the grid point ending with an op move. Computing $L$ corresponds to finding, for each $c, k$, and $o p$, the furthest position along diagonal $k$ that can be reached by a path of cost $c$ ending with op. For the example giving rise to Table IV, $L(13,-1$, move $)=5$ since the last 13 in a move position on diagonal -1 occurs in row 5 of the $C$ matrix. Also, $L(7,-1$, move $)=N O N E$ because a script containing a move must cost at least 8 .

With the general cost functions of the row-replacement problem, there can be paths of some cost $c$ reaching some diagonal $k$ and ending with some operation op even if no $c$ appears in an op entry of diagonal $k$ of the $C$ matrix. For such a $c, k$, and $o p, L(c, k, o p)$ is defined but (unlike the case where all costs are 1) cannot be characterized as the last row where a $c$ occurs in a diagonal of $C$. For example suppose deleting or inserting $n$ symbols costs $2 n$ and replacing $n$ symbols costs $3 n$. The replace entries on diagonal 0 are $0,3,6, \ldots$, but $L(7,0$, replace $)=$ 2 because the script 'Delete, Insert $b_{1}$, Replace by $b_{2}$ ' edits $A_{2}$ to $B_{2}$. However if $c$ occurs in an op position in diagonal $k$ of $C$, then $L(c, k, o p)$ is the row containing the last such $c$.

For values of $c$ in the order $1,2, \ldots$, the greedy algorithm computes $L(c, k, o p)$ for a relevant range of diagonals $k$ and all choices of op. The algorithm can stop when it reaches the first value of $c$ for which $L(c, N-M, o p)=M$ for some op because it has encountered the smallest cost, $c$, for which $A_{M}=A$ can be edited into $B_{M+(N-M)}=B$. We first show how to compute $L(c, k, o p)$ from previously computed values. General scripts are then discussed. Finally the complete algorithm is presented and its complexity analyzed.

The greedy algorithm presented below rests on three additional but realistic assumptions about the cost parameters:

(A1) $\operatorname{perchar}($ delete) $>0$ and perchar (insert) $>0$

(A2) perchar (replace) $>0$

(A3) $\operatorname{perchar}($ move $)=0$ and $\operatorname{startup}($ move $)>0$

These assumptions are needed for two reasons. First they permit the formulation of computable recurrences for the $L$ values of cost $c$ from the $L$ values of strictly smaller cost. Specifically, Assumptions A1 and A2 guarantee that best $(c, k, o p)$ (defined below) is a function of $L$ values of cost strictly less than $c$ and A3 guarantees the same property for mbest $(c, k)$. The second reason is to ensure that the band of diagonals considered has width not exceeding $2 c+1$. Specifically, A1 guarantees that neither upper $(c)$ nor lower (c) (defined below) exceeds $c$.

For the greedy method to be efficient, Assumption A1 is essential. Without it the approach produces an $O(M N)$ algorithm, no better than dynamic programming, because every diagonal must be considered. However Assumptions A2 and $\mathrm{A} 3$ are not critical. A3 can be changed to perchar (move) $>0$ and A2 can be changed to perchar (replace) $=0$ and startup (replace) $>0$, without changing the feasibility or time complexity of the greedy approach. Nonetheless either the assumptions or their alternates must be chosen a priori because the choice affects the logic of the algorithm. The most questionable of the assumptions is perchar(delete) $>0$, since some terminals support a fixed length "delete $n$ symbols" command. However many of these devices disguise a greater-than-zero 
perchar cost by requiring the transmission of a number of "pad characters" proportional to $n$.

Fix op $\in O P S$ and integers $c>0$ and $k$, and suppose $L(C, K, \theta)$ has been determined for all $C<c$ and all $K$ and $\theta$. Let firstop (op) be the cost of a first operation in an edit script:

$$
\text { firstop }(o p)=\operatorname{startup}(o p)+\operatorname{perchar}(o p)
$$

Then $L(c, k, o p)$ is computed by:

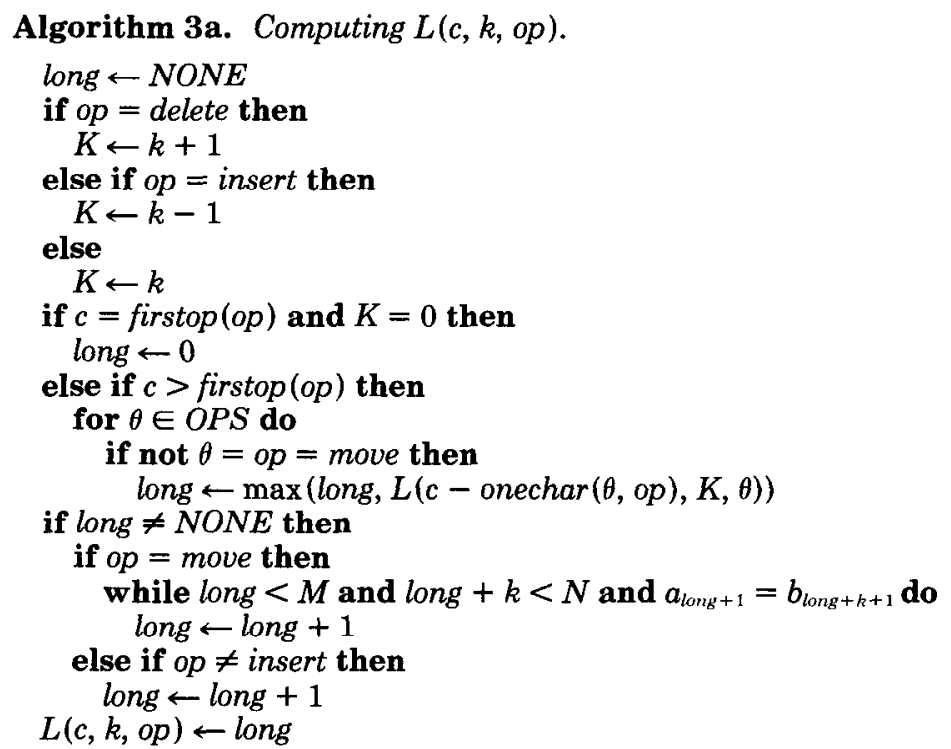

The only script of cost firstop (op) ending with op consists of a single op command. A single delete command edits $A_{1}$ to $B_{0}$, a single replace command edits $A_{1}$ to $B_{1}$, and a single insert command edits $A_{0}$ to $B_{1}$. 'The best single move command edits the longest common prefix of $A$ and $B$ into itself. These observations lead to the equations:

$$
\begin{aligned}
& L(\text { firstop }(\text { delete }),-1, \text { delete })=1 \\
& L(\text { firstop }(\text { insert }), 1, \text { insert })=0 \\
& L(\text { firstop }(\text { replace }), 0, \text { replace })=1 \\
& L(\text { firstop }(\text { move }), 0, \text { move })=\max \left(i \| A_{i}=B_{i}\right)
\end{aligned}
$$

For any other case with $c \leq$ firstop $(o p), L(c, k, o p)=N O N E$. These observations verify the correctness of Algorithm 3 a for small values of $c$.

For $c>$ firstop (delete), consider long $=L(c, k$, delete), the length of the longest prefix $A_{\text {long }}$ transformable to $B_{\text {long }+k}$ by a cost-c script ending with delete. Define:

$$
\operatorname{best}(c, k, o p)=\max _{\theta \in O P S}(L(c-\operatorname{onechar}(\theta, o p), k, \theta))
$$

Suppose that long $\neq N O N E$ and that stripping the final delete from a corresponding script leaves a script ending with operation $\theta$. The truncated script costs c-onechar $\left(\theta\right.$, delete) and converts $A_{\text {long-1 }}$ to $B_{\text {long }+k}$. Thus long $-1 \leq L$ (c-onechar $(\theta$, delete $), k+1, \theta) \leq$ best $(c, k+1$, delete $)$. We claim that long $-1=\operatorname{best}(c, k+1$, ACM Transactions on Programming Languages and Systems, Vol. 11, No. 1, January 1989. 
delete). Suppose there were a longer prefix, $A_{\text {long'-1 }}$ with long' $>$ long, that could be transformed to $B_{\text {long' }+k}$ with a script costing $c$-onechar $\left(\theta^{\prime}\right.$, delete $)$ and ending with operation $\theta^{\prime}$. Then appending a delete command gives a cost-c script that transforms $A_{\text {long }}$ to $B_{\text {long' }+k}$, contradicting the maximality of long. Thus $L(c, k$, delete $)=\operatorname{best}(c, k+1$, delete $)+1$ if it exists. Intuitively, to reach as far as possible down diagonal $k$ at $\operatorname{cost} c$, "greedily" append a delete move to a furthestreaching path of cost $c-$ onechar $(\theta$, delete $)$ ending in diagonal $k+1$. Finally observe that $L(c, k$, delete $)=N O N E$ if and only if $L(c-o$ onechar $(\theta$, delete $)$, $k+1, \theta)=N O N E$ for all $\theta \in O P S$. Similar reasoning for replace and insert leads to the following recurrences.

$$
\begin{aligned}
L(c, k, \text { delete })= & \text { if best }(c, k+1, \text { delete }) \neq N O N E \\
& \text { then best }(c, k+1, \text { delete })+1 \text { else NONE } \\
L(c, k, \text { replace })= & \text { if best }(c, k, \text { replace }) \neq N O N E \\
& \text { then best }(c, k, \text { replace })+1 \text { else } N O N E \\
L(c, k, \text { insert })= & \text { if best }(c, k-1, \text { insert }) \neq N O N E \\
& \text { then best }(c, k-1, \text { insert }) \text { else } N O N E
\end{aligned}
$$

For a concrete example, consider computing $L(19,-1$, insert $)$ for the ANSIstandard model, $A=a b c a b b a$ and $B=c b a b a c$ (Table IV of Section 3). We seek the longest prefix $A_{i}$ that can be transformed into $B_{i-2}$ at cost 19 - onechar $(\theta$, insert) for some $\theta$, since appending an insert command to a corresponding script gives a script that transforms $A_{i}$ to $B_{i-1}$ at cost $19 . L(10,-2$, delete $)=$ $L(10,-2$, replace $)=6$, so taking $\theta$ to be delete or replace shows that $A_{6}$ can be edited to $B_{5}$ at cost 19 . Trying $\theta=$ move is fruitless, since $L(10,-2$, move $)=$ NONE. Extending the script for $L(18,-2$, insert $)=3$ obtains a script for transforming $A_{3}$ to $B_{2}$. Thus, $L(19,-1$, insert $)$ is 6 .

Computing $L(c, k$, move) requires a different approach since perchar(move) equals zero. For $c>$ firstop (move), consider long $=L(c, k$, move), the length of the longest prefix $A_{\text {long }}$ transformable to $B_{\text {long }+k}$ by a cost-c script ending with move. If there are several such scripts, consider one whose final move command moves right the fewest columns. Let:

$$
\operatorname{mbest}(c, k)=\max _{\theta \neq \text { move }}(L(c \text {-onechar }(\theta, \text { move }), k, \theta))
$$

Suppose that long $\neq N O N E$ and that stripping the final move from a corresponding script leaves a script ending with operation $\theta$. Note that $\theta \neq$ move since two consecutive moves can be economized into one. The truncated script costs $c$-onechar $(\theta$, move $)$ and converts $A_{\text {long-m }}$ to $B_{\text {long-m+k }}$, where $m$ is as small as possible. Thus long $-m \leq L(c-\operatorname{onechar}(\theta$, move $), k, \theta) \leq m b e s t(c, k)$ and $a_{\text {long-m+1 }}$ $\cdots a_{\text {long }}=b_{\text {long-m+k+1}} \cdots b_{\text {long }+k}$. We claim that long $-m=m b e s t(c, k)$. Suppose there were a longer prefix, $A_{\text {long- } m^{\prime}}$ with $m^{\prime}<m$, that could be transformed to $B_{\text {long- } m^{\prime}+k}$ by a script costing $c$-onechar $\left(\theta^{\prime}\right.$, move $)$ and ending with operation $\theta^{\prime}$. If $m^{\prime} \geq 0$, then appending a move command that moves the cursor $m^{\prime}$ columns to the right gives a cost-c script that transforms $A_{\text {long' }}$ to $B_{\text {long }}+k$, contradicting the minimality of $m$. If $m^{\prime}<0$, then appending a move command that moves zero columns to the right gives a cost-c script that transforms 
$A_{\text {long }-m^{\prime}}$ to $B_{l o n g-m^{\prime}+k}$, contradicting the maximality of long. This reasoning justifies the recurrence:

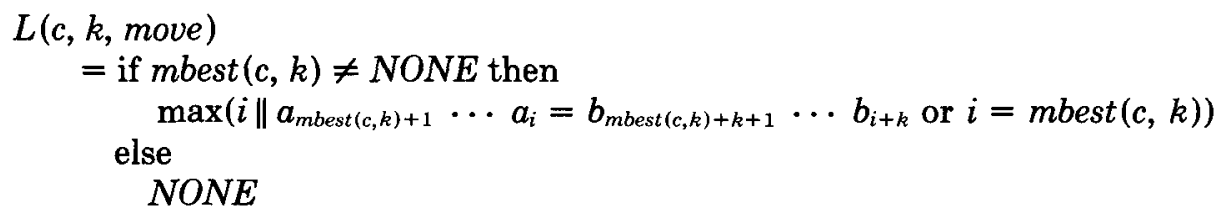

As the value of $c$ is increased, computed values may satisfy $L(c, k, o p)>M$ or $L(c, k, o p)+k>N$. This happens because the commands delete, replace, insert, and move-zero-columns-right are effective regardless of the characters actually in $A$ or $B$. Thus such an entry indicates that any supersequence of $A$ can be converted to a prefix of $B$ or that any prefix of $A$ can be transformed into a supersequence of $B$ at cost $c$. Such entries could be eliminated by an additional boundary check but this is unnecessary since they cannot contribute to the optimal solution.

Now turn attention to general row-replacement scripts. Since the algorithm's goal is to terminate as soon as the optimal value of $c$ is reached, the additional features of general scripts cannot be handled in a postpass, as with the dynamic programming algorithm. Instead, checks for general cost-c scripts are "folded" into the computation of $L$-values.

First consider removal of a final move operation. Before starting the computation of $L$, the longest common suffix of $A$ and $B$ is identified by setting:

$$
\lim =\min \left(i \| a_{i+1} \cdots a_{M}=b_{i+(N-M)+1} \cdots b_{N} \text { or } i=M\right)
$$

Pictorially this step finds a line segment beginning at the lower right corner of the grid and extending toward the upper left until the row and column labels disagree. If long $=L(c, N-M, o p) \geq \lim$ for some operation op then there is a cost-c script converting $A_{\text {long }}$ to $B_{\text {long }+(N-M)}$ and $a_{\text {long }+1} \cdots a_{M}=b_{\text {long }+(N-M)+1} \cdots$ $b_{N}$. This script transforms $A$ to $B$ and leaves the cursor on column long +1 . For general edit scripts the algorithm terminates upon encountering a value of $c$ for which long $\geq$ lim.

As explained in Section 3, if clear commands are permitted, then it suffices to consider a single clear at the end of the script. If $L(c, k, o p)=N-k$ for some $k>N-M$, then there is a cost-c script converting $A_{N-k}$ to $B$ where $N-k<M$. Appending a clear operation gives a script that edits $A$ into $B$ of cost $c+$ onechar (op, clear $)+(k-(N-M)-1) \times$ perchar (clear). The preceding argument assumes the clear operation is appended to a nonempty script, i.e., $c>0$. In the special case where $N=0$, a single clear edits $A$ into $B$ at cost startup(clear $)+M$ $\times$ perchar(clear). As scripts editing $A$ into $B$ and ending with clear are detected, their minimum cost is recorded in a variable Clim. If the loop variable $c$ reaches this value, the algorithm terminates and reports that the minimum rowreplacement cost is Clim. If an optimal script editing $A$ to $B$ ends with clear, it will be discovered because removing the clear from at least one such script leaves a script that edits a longest prefix of $A$ into $B$.

Handling overwrites beyond $A$ is the analog of final clear operations. If $L(c, k, o p)=M$ for some $k<N-M$, then there is a cost-c script converting $A_{M}$ to $B_{M+k}$ where $M+k<N$. Appending $N-(M+k)$ replace operations 
gives a script converting $A$ to $B$ of cost $c+$ onechar(op, replace) + $((N-M)-k-1) \times$ perchar (clear). In the special case where $M=0, N$ replace commands edit $A$ into $B$ at cost startup (replace) $+N \times$ perchar (replace). It is sufficient to let the variable Clim above also record the minimum cost of these overwrite-terminated scripts as each is detected.

Up to this point it has been assumed that for each value $c, L(c, k, o p)$ is computed for the entire range of diagonals, $k \in[-M, N]$. A central advantage of the greedy approach is that only $O(c) L$-values need to be computed since the remainder are easily inferred to be NONE. Let:

$$
\begin{aligned}
& \text { lower }(c)=\max \left(\frac{c-\text { startup }(\text { delete })}{\text { perchar }(\text { delete })}, 0\right) \\
& \text { upper }(c)=\max \left(\frac{c-\operatorname{startup}(\text { insert })}{\text { perchar }(\text { insert })}, 0\right)
\end{aligned}
$$

If a cost-c restricted replacement script transforms $A_{i}$ into $B_{i+k}$ where $k>0$, then it must insert at least $k$ characters. Thus $c \geq \operatorname{startup}($ insert $)+k \times$ perchar (insert) because at best the $k$ insert commands are contiguous. Rearranging the inequality gives the relationship $k \leq u p p e r(c)$. Arguing similarly about the case where $k<0$ shows that $k \geq-$ lower $(c)$ and leads to the fact:

$$
\text { If } k \notin[-\operatorname{lower}(c), \text { upper }(c)] \text { then } L(c, k, o p)=\text { NONE. }
$$

The algorithm uses this fact in two ways. First, for each value of $c$ it only computes $L(c, k, o p)$ for $k \in[-\operatorname{lower}(c)$, upper $(c)]$. Second, when referencing previously computed values, $L(C, K, \theta)$, in the innermost loop, it infers that the value is $N O N E$ if $K \notin[-\operatorname{lower}(C)$, upper $(C)]$.

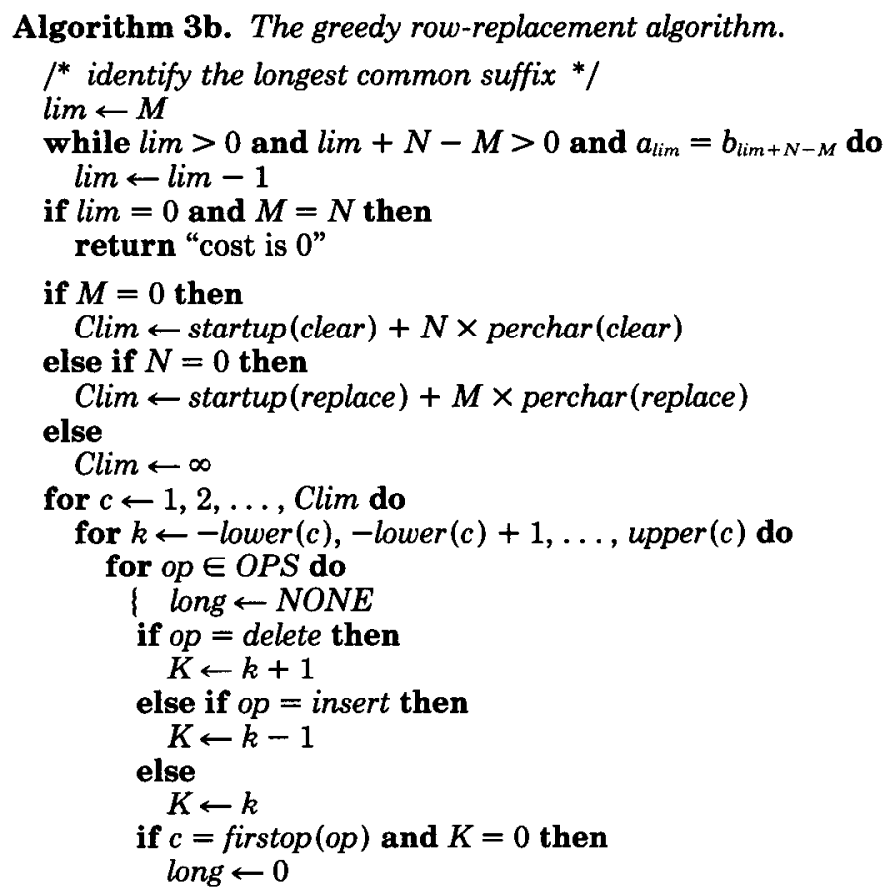

ACM Transactions on Programming Languages and Systems, Vol. 11, No. 1, January 1989. 


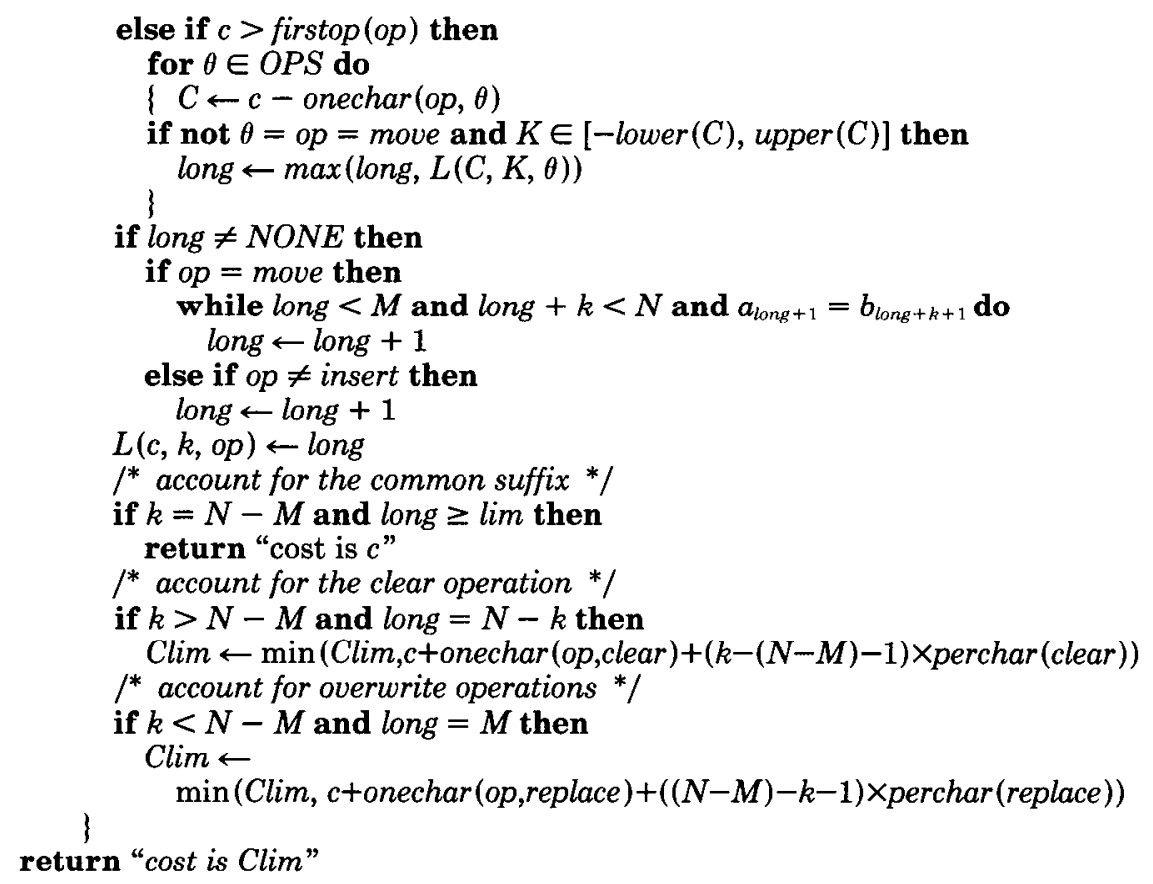

Let Cost be the optimal cost of replacing row $A$ with row $B$ of lengths $M$ and $N$, respectively. The greedy algorithm runs in time $O($ Cost $\times(M+N))$. Identifying the longest common suffix takes only $O(M+N)$ time. The code within the triply nested loops, 'for $c$, for $k$, for op', is repeated $\Theta\left(\right.$ Cost $\left.^{2}\right)$ times because the 'for c' loop is repeated Cost times, the 'for $k$ ' loop is repeated upper (c) + lower (c) $+1 \leq 2 c+1$ times (by Assumption A1), and the 'for op' loop is repeated four times. With the exception of the innermost while loop, the code within the three for loops takes $O(1)$ time. Thus all portions of the algorithm except for this while loop consume $O\left(M+N+\right.$ Cost $\left.^{2}\right)$ time.

To account for the time taken by the innermost while loop, charge each $O(1)$ iteration to the grid entry (long +1 , long $+k+1$ ) examined by the loop. We claim that a given entry is charged at most $\Delta=$ firstop (move) + firstop (replace) times. First observe that if $(i, i+k)$ is charged when computing $L(c, k$, move $)$, then the recurrence for $L(c, k$, move $)$ implies that $i \in[\operatorname{mbest}(c, k)+1$, $L(c, k$, move $)+1]$. Now suppose $(i, i+k)$ is first charged when computing $L(d, k$, move $)$. Then

mbest $(d+\Delta, k) \geq L(d+$ firstop $($ replace $), k$, replace $) \geq L(d, k$, move $)+1 \geq i$

Thus mbest $(C, k) \geq i$ for all $C \geq d+\Delta$. But then entry $(i, i+k)$ cannot be charged when computing $L(C, k$, move $)$ for such $C$, since the converse would imply mbest $(C, k)<i$. This proves the claim. The algorithm computes $L$-values only over the range of diagonals -lower(Cost) to upper (Cost) and there are less than $(2 \operatorname{Cost}+1) \min (M, N)$ grid points within this range. Since each grid point is charged $O(1)$ time (considering $\Delta$ to be a constant), it follows that $O$ (Cost $\times$ $(M+N))$ time is taken by the body of the innermost while loop. 
Reconstructing a script from the $O\left(\mathrm{Cost}^{2}\right) L$-values computed by the greedy algorithm is analogous to the postprocess described for the dynamic programming algorithm and requires only $O($ Cost ) additional time. However, hierarchical screen updating methods [6] require just the costs of row replacements when evaluating interline editing strategies. These costs can be computed in only $O($ Cost $)$ space as follows. Observe that the value of $L(c, k, o p)$ depends only on values $L(C, K, \theta)$ for which $C \geq c-M O P$ where $M O P=\max _{o p \in O P S}$ ( firstop(op)). Thus the cost-only algorithm need only retain the $L$-values of the most recent $M O P$ repetitions of the outer 'for $c$ ' loop and this requires only $O($ Cost $)$ space (considering $M O P$ to be a constant).

\section{DISCUSSION AND OPEN PROBLEMS}

Algorithmic Variations. Algorithms 2a, 2b, and $3 \mathrm{~b}$ can be extended in several ways. Of the two, the dynamic programming approach is more 'robust' in the sense of extending easily to certain generalizations of the problem. For example, like Algorithm 1a of Wagner and Fischer, Algorithms 2a and 2b work without change for arbitrary real costs. On the other hand, Algorithm 3b, like Algorithm $1 \mathrm{~b}$, requires integer costs, and efficiency considerations demand that perchar (delete) and perchar (insert) be positive.

Both algorithms can be made more space-efficient. To construct an optimal script, Algorithms 2a, 2b, and 3b, as presented, require retention of $O(M N)$ and $O\left(C_{0 s t^{2}}\right)$ arrays $C$ and $L$, respectively. If Algorithm 2b's postpass checks are integrated into Algorithm 2a, as was done in Algorithm 3b, then Hirschberg's divide and conquer technique [9] computes the replacement script in only linear space. Most of the details are explained in [16], which gives a linear-space sequence comparison algorithm for block indels with affine costs. Similarly Myers' approach [14] for computing an edit script in linear space can be adapted for Algorithm 3b. However in both cases the necessary adaptations are complex and probably unnecessary, given the typical sizes of row replacement problems that arise with screen editors.

Efficiency Considerations. An editor's screen-updating module must be efficient. As text is typed, the module is invoked with every keystroke. With the algorithms developed in this paper, efficiency considerations are paramount. Tests under realistic conditions favor the greedy approach over dynamic programming, but a straightforward implementation of Algorithm 3b may be impractically slow. Fortunately both algorithmic (high-level) and implementation (low-level) efficiency gains are possible with the greedy algorithm.

At the algorithmic level, the screen operations op with startup cost 0 can be lumped together since onechar $(\theta, o p)$ does not vary with $\theta$. Thus, with ANSIstandard terminals, the restricted replacement scripts can be classified: (1) ends with insert, (2) ends with move, and (3) ends with delete or replace. That is, entries for delete and replace are coalesced into a single entry delrep for which $L(c, k$, delrep $)=\min (L(c, k$, delete $), L(c, k$, replace $))$. This reduces space requirements and the number of executions of the innermost 'for op $\in$ OPS' loop by the factor $3 / 4$. 
The innermost while loop treating the zero perchar cost of move commands frequently visits a grid point as many as firstop (move) + firstop (replace) times. This can be reduced to one by maintaining a vector, $\operatorname{Far}(k)$, equal to the maximum of $L(d, k$, move $)$ for all $d$ less than the current $c$ of the outermost loop. Initially set $\operatorname{Far}(k)$ to NONE for all $k$ and change Algorithm $3 \mathrm{a}$ as follows.

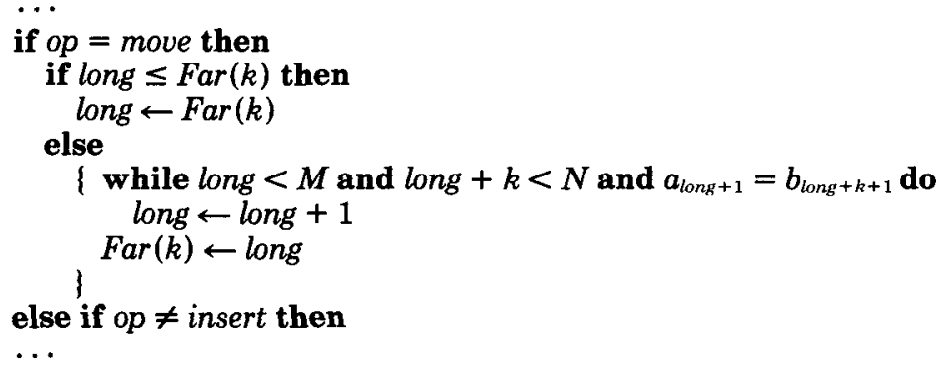

Essentially, mbest $(c, k)$ is nondecreasing in $c$ and so using $\operatorname{Far}(k)$ prevents the rediscovery of a sequence of zero-cost diagonal moves.

At the implementation level a number of code transformations are possible. The test ' $K \in[-$ lower $(C)$, lower $(C)]$ ' can be avoided within the inner loop by explicitly setting $L(c, k, o p)$ to NONE for $k \in[-\operatorname{lower}(c+M O P),-\operatorname{lower}(c)-1]$ $\cup[\operatorname{upper}(c)+1$, upper $(c+M O P)]$. The 'for op' and 'for $\theta$ ' loops can be completely unrolled and the resulting code specialized by eliminating the now unnecessary tests on op and $\theta$. Similarly the 'for $c$ ' outer loop can be broken into a loop for values less than or equal to $M O P$ and one for greater values. In the second copy of the loop, the code is specialized by eliminating all tests on $c$. By far the greatest low-level efficiency gains can be made by replacing the triple subscripting for $L(c, k, o p)$ by pointer manipulations. In effect, this involves hand-coding of the strength-reduction optimizations that the compiler cannot detect.

Empirical Results. Nine row-replacement problems were solved by the programs:

implementation of the algorithm used by the $s$ editor $[10, \mathrm{pp} .290-$ 298]

Dyna straightforward implementation of Algorithms 2a and 2b

Greedy straightforward implementation of Algorithm $3 \mathrm{~b}$

Hand implementation of Algorithm $3 \mathrm{~b}$ optimized as above

Separate measurements were taken for the ANSI and the IBM 3101 cost parameters, since cost parameters determine which scripts are optimal and affect the performance of the greedy method. Table $\mathrm{V}$ summarizes the results. Row labels have the following interpretations:

$M: N$

Script lengths of the input rows $A$ and $B$.

replacement script generated by $S$. Edit commands are indicated by letters $C$ (Clear), $D$ (Delete), $I$ (Insert), $M$ (Move) and $R$ (Replace). For example, ' $R{ }^{2} D M I$ ' denotes a script of the form 'Replace, Replace, Delete, Move, Insert'. 


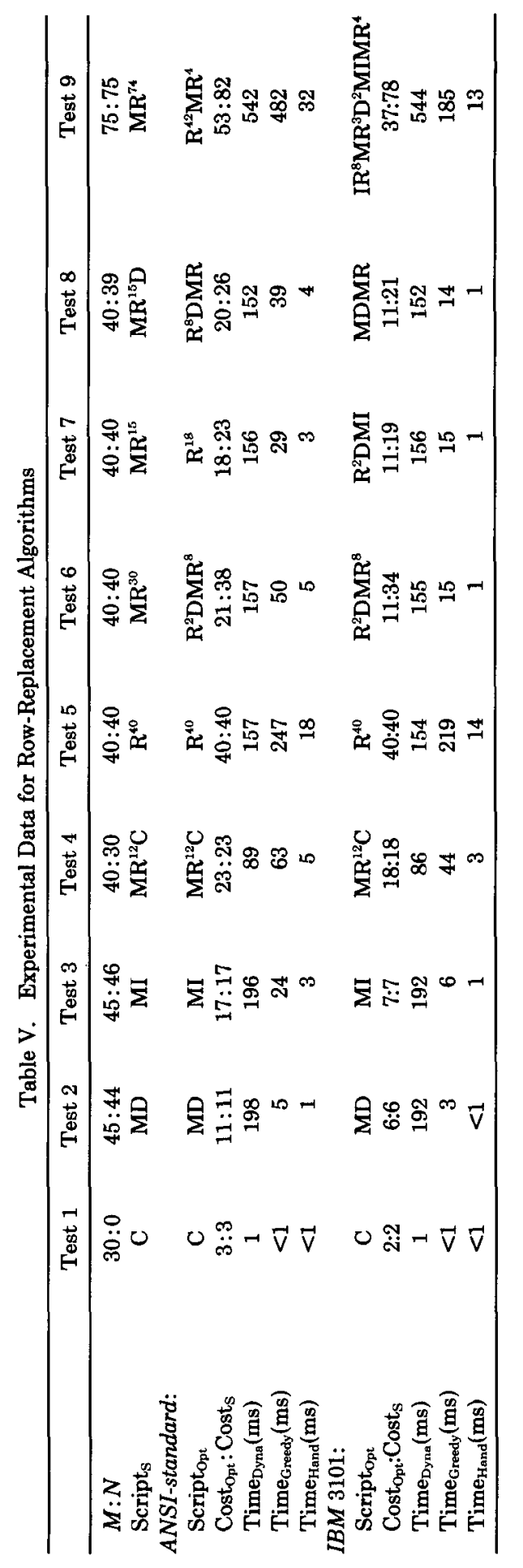


Script $t_{O p t} \quad$ optimal replacement script.

Cost $_{\text {Opt }}:$ Cost $_{S} \quad$ length of the optimal script and of the script generated by $S$.

Time $_{\text {Dyna }}(m s) \quad$ execution time of Dyna in milliseconds. The procedures were written in $\mathrm{C}$ and run on a DEC 8600 under the UNIX operating system.

Time $_{\text {Greedy }}(m s) \quad$ execution time of Greedy in milliseconds.

Time $_{\text {Hand }}(\mathrm{ms}) \quad$ execution time of Hand in milliseconds.

A number of interesting facts can be extracted from this data, but two points deserve explicit mention. First, Greedy was more efficient than Dyna, especially for the 3101. Execution time for the greedy method depends on terminal cost parameters and improves with the 3101's shorter escape sequences. Dyna's execution is essentially independent of these costs. Moreover, hand optimization sped up the greedy algorithm by a factor of 12 for the ANSI model and 15 for the 3101.

Table $\mathrm{V}$ also indicates conditions where a hand-optimized greedy program can outperform a simple row-replacement procedure. A method's performance is determined by the time to compute the script and transmit it to the screen. Assuming that it takes a millisecond to transmit a character (e.g., at $960 \mathrm{n}$ baud), Hand was superior to $S$ whenever $S$ failed to generate an optimal script. This occurred when the optimal script contained a noninitial move command. The superiority of Hand over $S$ benefited from the 3101's economical move. (Table V was simplified by omitting $S$ 's execution times, which were always less than a millisecond, and by giving a single line of $S$ 's scripts, which happened to coincide for the two cost models on Tests 1-9.)

Open Problems and Future Work. The key assumptions in this paper are that the cursor begins in column 1 and that spilling characters off the end of a row is not a concern. While a script produced by our algorithms can be transformed into one that takes into account the current cursor location and avoids a spill, the transformed script is not guaranteed to be optimal. It appears very difficult, but still solvable in polynomial time, to compute an optimal script that takes into account these factors. Designing an efficient algorithm for this problem is a subject of current research.

Row replacement is just a subproblem in the ultimate goal of building an efficient, but autonomous, screen manager that optimally updates the entire screen. Optimality is sacrificed by the natural two-level approach that decides which lines to delete, insert, and replace at the top level, and that uses row replacement to appraise and perform replacements at the bottom level. Moreover, there are natural asymmetries between intra- and inter-row edits that resist a homogeneous approach. For example, unlike character insertion, the cost of a row insertion command is "symbol-dependent" since its cost is a function of the length of the row itself. This prevents the greedy algorithm of Section 4 from being applied to interline edits. It remains to be seen whether an optimal "screen replacement" algorithm of satisfactory efficiency can be developed.

The change from terminals and serial communications to workstations and high-speed networks does not make the screen-update problem disappear, though different techniques are needed to minimize the cost of bitblt operations [8]. Pike ACM Transactions on Programming Languages and Systems, Vol. 11, No. 1, January 1989. 
[17] discusses a simple approach for screen updating by a text editor, while [3] treats display-updating problems in computer animation. However, the problem of optimally updating a bitmap display is not well understood.

What is the most general class of sequence comparison problems that can be solved with the greedy approach? Algorithm $3 \mathrm{~b}$ solves, as a special case, the problem for block indels with affine gap penalties, which is important in molecular biology. The algorithm can be extended to handle symbol-dependent replacement costs, which are used for comparing proteins. However, the exact limits of the greedy algorithm remain to be determined.

\section{ACKNOWLEDGMENTS}

The authors thank the referees for numerous suggestions that improved this presentation.

\section{REFERENCES}

1. ARNOLD, K. C. R. C. Screen updating and cursor movement optimization: a library package. In UNIX Programmer's Manual: Supplementary Documents (4.2BSD), University of California, Berkeley, 1984.

2. Barach, D., TaEnzer, D., AND Wells, R. The design of the PEN video editor display module. In Proceedings of the ACM Symposium on Text Manipulation (Portland, Ore., June 8-10, 1981). SIGPLAN Not. 16, 6 (1981), 130-136.

3. Denber, M. J., AND TURner, P. M. A differential compiler for computer animation. Comput. Graph. 20, 4 (1986), 21-27.

4. FiCKETT, J. W. Fast optimal alignment. Nucleic Acids Res. 12, 1 (1984), 175-179.

5. Fitch, W. M., AND SMITh, T. F. Optimal sequence alignments. In Proceedings National Academy of Science USA 80 (1983), 1382-1386.

6. GosLing, J. A redisplay algorithm. In Proceedings of the ACM Symposium on Text Manipulation (Portland, Ore., June 8-10, 1981). SIGPLAN Not. 16, 6 (1981), 123-129.

7. Gотон, O. An improved algorithm for matching biological sequences. J. Molec. Biol. 162 (1982), 705-708.

8. GuiBas, L. J., AND Stolfi, J. A language for bitmap manipulation. ACM Trans. Graph. 1, 3 (1982), 191-214.

9. HirschBerG, D. S. A linear space algorithm for computing maximal common subsequences. Commun. ACM 18, 6 (1975), 341-343.

10. Miller, W. A Software Tools Sampler. Prentice-Hall, Englewood Cliffs, N.J., 1987.

11. Miller, W., AND Myers, E. W. A file comparison program. Softw. Pract. Exper. 15, 11 (1985), 1025-41.

12. Miller, W., AND Myers, E. W. Sequence comparison with concave weighting functions. Bull. Math. Biol. 50, 2 (1988), 97-120.

13. Miller, W., AND Myers, E. W. A simple row replacement method. Softw. Pract. Exper. 18, 7 (1988), 597-612.

14. MYERS, E. W. An $O(N D)$ difference algorithm and its variants. Algorithmica 2,1 (1986), 251-266.

15. MyerS, E. W. Incremental alignment algorithms and their applications. To appear in SIAM J. Comput. See also TR86-22, Dept. of Computer Science, Univ. of Arizona, Tucson.

16. Myers, E. W., AND Miller, W. Optimal alignments in linear space. Comput. App. Biosci. 4, 1 (1988), 11-17.

17. PIKE, R. The text editor sam. Softw. Pract. Exper. 17, 11 (1987), 813-845.

18. Stallman, R. M. EMACS-the extensible, customizable self-documenting display editor. In Proceedings of the ACM Symposium on Text Manipulation (Portland, Ore., June 8-10, 1981). SIGPLAN Not. 16, 6 (1981), 147-157.

19. UKKonEN, E. Algorithms for approximate string matching. Inf. Contr. 64, 1-3 (1985), 100-118. 
20. WAGNeR, R. A., AND Fischer, M. J. The string-to-string correction problem. J. $A C M 21,1$ (1974), 168-173.

21. Waterman, M. S. General methods for sequence comparisons. Bull. Math. Biol. 44, 4 (1984), 473-500.

22. Waterman, M. S., Smith, T. F., AND Beyer, W. A. Some biological sequence metrics. Advances in Math. 20 (1976), 367-387.

23. Weiser, M. CWSH: The windowing shell of the Maryland Window System. Softw. Pract. Exper. 15, 5 (1985), 515-519.

24. Woon, S. R. 7-the 95\% program editor. In Proceedings of the ACM Symposium on Text Manipulation (Portland, Ore., June 8-10, 1981). SIGPLAN Not. 16, 6 (1981), 1-7.

Received July 1986; revised November 1987 and August 1988; accepted August 1988 